\title{
VALDITYY OF AN INERTIILL MEASUREMENT UNIT SYSTEM TO ASSESS LOWER-LIMB KINEMATICS DURING A MAXIMAL LINEAR DECELERATION
}

\author{
Alastair R. Jordan, ${ }^{1, A, B, C, D}$ Howie J. Carson, ${ }^{2, A, B, ~ C, ~ D ~ B r e t t ~ W i l k i e,, ~ C, ~ D ~}$ \\ Damian J. Harper ${ }^{3, A, B, C, D}$ \\ ${ }^{1}$ School of Science Technology and Health, York St John University, Haxby Road Sports Park, York, YO31 8TA, United Kingdom \\ ${ }^{2}$ Institute for Sport, Physical Education and Health Sciences, Moray House School of Education and Sport, The University of Edinburgh, United \\ Kingdom \\ ${ }^{3}$ Institute of Coaching and Performance, School of Sport and Health Sciences, University of Central Lancashire, United Kingdom

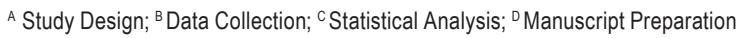

Address for corpespondence:

Alastair R. Jordan

School of Science Technology and Health, York St John University

Haxby Road Sports Park, Y031 8TA, United Kingdom

Email: a.jordan1@yorksj.ac.uk

\begin{abstract}
Ahstract This study examined the validity of an inertial measurement unit system for measuring lower-limb joint kinematics during linear decelerations. A male team athlete (age 36 years, stature $1.75 \mathrm{~m}$, mass $80.0 \mathrm{~kg}$ ) performed multiple linear decelerations, following $20 \mathrm{~m}$ runs at $50 \%, 75 \%$ and $100 \%$ self-perceived effort. Inertial measurement unit sensors were strapped to lower-limb segments and retroreflective markers were adhered to the lower-limbs for 3D optical motion analysis. Ground contact time, foot to centre of mass displacement (foot-COM), peak and minimum angle, mean angular velocity and range of motion at the ankle, knee and hip during the contact phases of each deceleration were determined. Measures were valid if a very large correlation $(r \geq 0.7)$ and small bias (effect size $<0.6)$ were evident. Following $50 \%$ effort, ground contact time, foot-COM and most hip and knee kinematics were valid. Ground contact time, foot-COM and knee flexion velocity and range of motion were valid following $75 \%$ efforts. Ground contact time and knee flexion velocity were valid following $100 \%$ effort. Therefore, the inertial measurement unit system tested can be used to assess temporal-spatial parameters during a deceleration regardless of the preceding effort, and hip and knee kinematics following low intensity running.
\end{abstract}

Key WOrlls biomechanics, braking, IMU, stopping, Xsens

\section{Introduction}

An ability to decelerate is emerging as a critical component of sport performance (Harper, Jordan, Kiely, 2021; Harper, Kiely, 2018; Hewit, Cronin, Button, Hume, 2011). Analyses of team-based sports, such as soccer, have indicated as many as 430 lower intensity $\left(\leq-2{\left.\mathrm{~m} . \mathrm{s}^{-2}\right)}\right.$ decelerations (Mara, Thompson, Pumpa, Morgan, 2017) and approximately 43-54 high intensity decelerations $\left(\leq-3 \mathrm{~m} . \mathrm{s}^{-2}\right)$ during a match (Bloomfield, Poman, O'Donoghue, 2008; Dalen, Ingebrigtsen, Ettema, Hjelde, Wisloff, 2016; Russell et al., 2016). Deceleration underpins many of the 
hundreds of changes in movement patterns observed in a match, including stopping to evade an opponent or collision (Hewit et al., 2011) and precedes a change of direction (Chaouachi et al., 2012; Dos'Santos, Thomas, Comfort, Jones, 2018; Green, Blake, Caulfield, 2011).

Faster deceleration requires the centre of mass to be positioned more posterior to the lead foot at ground contact, thereby maximising horizontal braking force application (Cesar, Sigward, 2015; Hewit et al., 2011). Upon contact with the ground, high eccentric loading of the lower-limbs results in rapid hip and knee flexion and dorsiflexion of the ankle to disperse the impact forces effectively (Nedergaard, Kersting, Lake, 2014; Spiteri et al., 2014). Furthermore, it has been suggested that a high proportion of non-contact lower-limb injuries, such as anterior cruciate ligament or posterior chain musculature, occur during the contact phase of deceleration when the knee is at or near full extension and then undergoes rapid eccentric knee flexion (Chaouachi et al., 2012; Cochrane, Lloyd, Buttfield, Seward, McGivern, 2007; Kovacs, Roetert, Ellenbecker, 2008). Consequently, execution of appropriate deceleration kinematic strategies is essential to maximising deceleration performance and minimising injury risk.

Deceleration technique can be assessed by 3-dimensional optical motion analysis (3DOMA) as the gold standard for movement analysis, however these camera-based systems are expensive, athletes must perform within the confines of a calibrated capture volume that is typically small and reflective markers can be obscured at critical moments during a dynamic performance. Inertial measurement units (IMU) consist of gyroscopes, magnetometers and accelerometers contained within unobtrusive lightweight units and could provide valid information on deceleration kinematics, without the drawbacks of 3DOMA. IMU systems are easier to set up and can be used in field-based environments where athletes are not constrained to a calibrated capture volume. A commonly researched IMU system is the Xsens Technologies B.V (Adesida, Papi, McGregor, 2019; Camomilla, Bergamini, Fantozzi, Vannozzi, 2018) and has been used to assess movement in a variety of functional movement tasks (Fleron, Ubbesen, Battistella, Dejtiar, Oliveira, 2019; Laudanski, Brouwer, Li, 2013; Zhang, Novak, Brouwer, 2013) and sporting contexts (Blair, Duthie, Robertson, Hopkins, Ball, 2018; Carson, Collins, Richards, 2014; Krüger, Edelmann-Nusser, 2009; Reenalda, Maartens, Homan, Buurke, 2016; Wouda et al., 2018).

Despite extensive usage of the Xsens IMU system, there has been limited attention to the validity of measures, particularly those associated with tasks involving whole body deceleration. Valid measures of performance are essential to provide coaches and athletes with valuable information on sport performance from injury prevention and performance enhancement perspectives. Xsens has been validated against 3DOMA and demonstrated very good - excellent validity for sagittal plane flexion and extension angles during walking, stair ascent and descent (Seel, Raisch, Schauer, 2014; Zhang et al., 2013) and treadmill running at different speeds (Wouda et al., 2018). Lower validity has been shown in frontal and transverse plane kinematics (Wouda et al., 2018; Zhang et al., 2013), whilst transitioning between acceleration and deceleration during walking (Fleron et al., 2019) and with faster lowerlimb segment velocities (Blair et al., 2018; Ferrari et al., 2010). Based on these findings, validity should be reported for speed and type of movement.

Currently, the validity of Xsens IMU systems for measuring lower-limb kinematics during deceleration from various running speeds is unknown. Therefore, the aim of this study was to assess the concurrent validity of lowerlimb joint kinematic measures from the Xsens IMU system compared with 3DOMA during linear decelerations from various running speeds. 


\section{Methods}

\section{Participant}

Following institutional ethical approval, a male competitive soccer player (age 36 years, stature $1.72 \mathrm{~m}$, mass $80.0 \mathrm{~kg}$ ) with 15 years previous playing experience provided written consent to participate in this study. The participant regularly took part in soccer training and moderate-to-vigorous intensity physical activity (3-5 times per week) lasting between 60-90 minutes per session and was, therefore, accustomed to acceleration and deceleration type activities. This study adopted a single-participant approach, which has been used in previous research by Buchheit, Gray, Morin (2015).

\section{Decelepation Task}

The participant performed a series of acceleration-deceleration ability tests (ADA) on one occasion. As described by Harper et al. (2021), the ADA consists of a $20 \mathrm{~m}$ run, a maximal linear deceleration after crossing the $20 \mathrm{~m}$ line and backpedal to the $20 \mathrm{~m}$ line (Figure 1). The backpedeal created a clear 'stop' event and indicated the end of the deceleration phase (Harper et al., 2021).

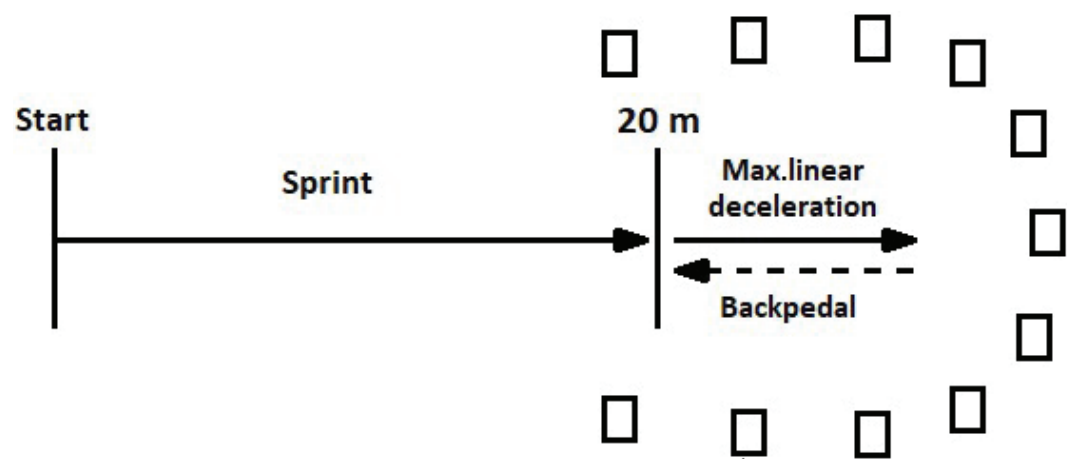

Figure 1. Acceleration-deceleration ability (ADA) test used to assess maximal linear deceleration. Symbol $\mathbf{Q}$ denotes position of 3D optical motion analysis cameras

To assess the validity of the Xsens IMU system when decelerating from various speeds, the participant performed $10 \times 20$ m runs at $50 \%, 75 \%$ and $100 \%$ self-perceived effort (30 trials in total) separated by $3-5$ minutes of rest. A minimum 3 min rest between each ADA trial was considered sufficient recovery for the participant and exceeded the durations and work:rest ratios suggested in previous research to achieve a rested state following similar short bouts of sprint activity (Abt, Siegler, Akubat, Castagna, 2011; Balsom, Seger, Sjödin, Ekblom, 1992; Coleman, 2001; Spencer, Bishop, Dawson, Goodman, 2005). A maximum 5 min recovery was permitted to check and address the equipment calibration. Xsens IMU system captured kinematics in the entire ADA whereas 3DOMA captured the deceleration phase only. 


\section{Inertial Measurement Unit System}

In accordance with manufacturer instructions, Xsens IMU sensors (MTx2, Xsens MVN Biomech, Xsens Technologies, The Netherlands) were strapped firmly to segments of the participant's lower-limbs, specifically at the feet (midpoint of the bridge of the foot), shanks (medial surface of the tibia), thighs (lateral aspect above the knee) and pelvis (midpoint of the sacrum). MVN Studio Pro software (Xsens Technologies, The Netherlands) was used to calibrate and track the IMUs $(240 \mathrm{~Hz})$ during the ADA. The calibration protocol began with the participant standing in the N-pose (arms neutral besides body) and a series of movements to orientate IMU sensors and functional axes of body segments. The participant's body height, arm span, foot size and anatomical landmarks of the greater trochanter (hip height from the ground), lateral femoral epicondyle (knee height from the ground), lateral malleolus (ankle height from the ground), left and right anterior superior iliac spine (hip width) were measured to estimate joint centres and segment dimensions of the model in MVN Studio Pro software.

\section{Optical Motion Analysis (3DOMA)}

Eleven infrared 3DOMA cameras (Oqus 300, Qualisys, Sweden) were positioned around the deceleration area (Figure 1). Qualisys Track Manager ${ }^{\mathrm{TM}}$ software (Qualisys, Sweden) was used to calibrate (average residuals of $<1 \mathrm{~mm}$ ) and track $(240 \mathrm{~Hz})$ spherical retroreflective markers (Qualisys, Sweden) adhered to the participant's skin or the Xsens elasticated suit (Figure 2).
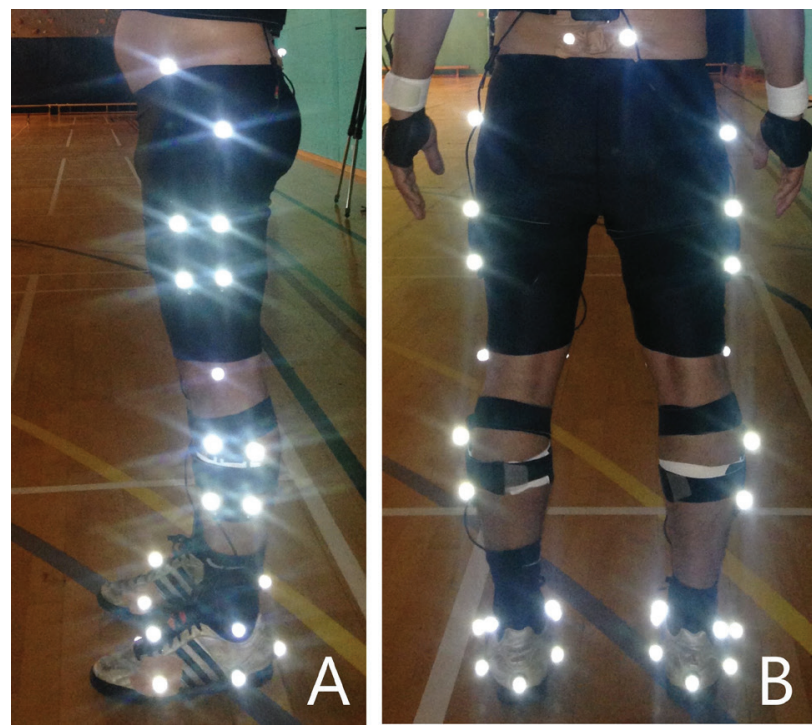

Figure 2. Sagittal (A) and posterior view (B) of the participant with Xsens IMU sensors strapped to lower-limb segments with retroreflective markers overlying anatomical landmarks and cluster markers strapped to lower-limb segments 
Retroreflective markers were positioned bilaterally on the anterior superior iliac spine, posterior superior iliac spine, greater trochanter, medial and lateral femoral epicondyle, medial and lateral malleoli, calcaneus and the superior aspect of the foot, plus the $1^{1 \text { st }}$ and $5^{\text {th }}$ metatarsal heads. Cluster markers (four markers on a rigid baseplate) were positioned on the lateral aspect of the thigh and shank and affixed firmly with compression wraps (Fabrifoam, Applied Technology International Ltd., Pennsylvania, USA). Following a static capture trial (from both the 3DOMA and Xsens IMU), the medial and lateral femoral epicondyles, medial and lateral malleoli and greater trochanter markers were removed prior to performing the ADA. A single reflective marker was placed on the $20 \mathrm{~m}$ line to signify the beginning of the deceleration phase of the ADA.

\section{Data Analysis}

3DOMA marker trajectories during deceleration phase were labelled in Qualisys Track Manager $^{\mathrm{TM}}$ software and exported to Visual 3D motion analysis software in c3d file format (C-Motion, Rockville, MD, USA) for further processing and analysis. Raw 3DOMA marker coordinate data were interpolated and filtered using a zero-lag fourth order low-pass Butterworth filter with a cut-off frequency of $30 \mathrm{~Hz}$ to remove clear high frequency noise in the signal (Nedergaard et al., 2014). The segment information (axes of rotation, segment dimensions and orientation) from the Xsens IMU data were also exported to c3d file format into Visual 3D software for analysis. The Xsens IMU data were filtered and processed by manufacturer software (MVN Studio Pro, Xsens Technologies, The Netherlands), therefore further filtering was not required in Visual 3D. Visual 3D created six-degrees of freedom multi-segmental models from the 3DOMA marker data and Xsens IMU data. Both systems were assigned joint centres defined by the medial and lateral markers on the proximal and distal aspects for each segment using the single frame of a static calibration trial (y-axis = anterior-posterior, $x$-axis $=$ medial-lateral and $z$-axis $=$ proximal-distal). Joint kinematics were calculated in flexion/extension using the distal segment relative to the local coordinate system of the proximal segment, using an $\mathrm{X}$ (flexion/extension), $\mathrm{Y}$ (medial/lateral), Z (axial) Cardan sequence.

Temporal-spatial and kinematic data were analysed for the deceleration phase only, defined as the first foot contact after the model COM passed the deceleration marker ( $20 \mathrm{~m}$ line) to the last ground contact prior to posterior movement of the model in the backpedal. Initial contacts were identified as the minimum vertical position of the calcaneus marker (heel strike) or the $5^{\text {th }}$ metatarsal marker (forefoot strike) (Cesar, Sigward, 2016). Toe off was identified at the onset of vertical displacement of the $5^{\text {th }}$ metatarsal (Cesar, Sigward, 2015). All initial contact and toe off events, for both 3DOMA and Xsens IMU, were verified by visual inspection. Temporal-spatial parameters of interest were ground contact time (GCT) and anterior/posterior foot to COM displacement. Sagittal plane kinematic variables of interest during trials at 50\%,75\% and 100\% effort included peak and minimum joint angles, average angular velocity (flexion MAV and extension MAV) and range of motion (ROM) at the hip, knee and ankle during contact phase. Approach velocity into the deceleration phase was determined in 50\%, $75 \%$ and $100 \%$ efforts using the velocity of the COM in the 3DOMA.

\section{Statistical Analysis}

Mean and standard deviation (SD) of all variables during trials at 50\%,75\% and 100\% effort were calculated from 3DOMA and Xsens IMU system. The agreement between Xsens and 3DOMA (criterion measure) measures was assessed using a spreadsheet by Hopkins (2017). The spreadsheet calculated Pearson's correlation coefficient $(r)$, mean difference $( \pm 90 \%$ confidence limits $(C L))$ and effect size $(E S, \pm 90 \% C L)$ and raw typical error of estimate 
(TEE, $\pm 90 \% \mathrm{CL}$ ) to assess strength of relationships, systematic bias and typical error between measurements, respectively. The magnitude of the Pearson's $r$ was interpreted as $\leq 0.1$, trivial; $>0.1-0.3$, small; $>0.3-0.5$, moderate; $>0.5-0.7$, large; $>0.7-0.9$, very large; and $>0.9-1.0$, almost perfect (Hopkins, 2002). Thresholds for ES were $<0.2$, trivial; 0.2-0.6, small; 0.6-1.2, moderate; 1.2-2.0, large; $2-4$, very large and $>4$ extremely large (Hopkins, 2002). Temporal-spatial and kinematic measures were considered valid if a very large correlation $(r \geq 0.7)$ and small bias (ES < 0.6 ) were evident. Differences in approach velocity between $50 \%, 75 \%$ and $100 \%$ efforts were determined by within-subjects repeated measures ANOVA with Bonferroni pairwise comparisons (Jamovi version 0.9, Jamovi Project, Amsterdam, The Netherlands).

\section{Results}

The participant's perceptions of effort on the approach to the deceleration resulted in significant differences in approach velocities $(p<0.05)$. As perceived effort increased from $50 \%, 75 \%$ and $100 \%$, approach velocity

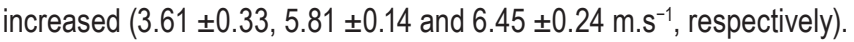

Table 1. Mean (standard deviation) of temporal-spatial parameters (Ground Contact Time (GCT) and anterior foot to centre of mass displacement (Foot-COM) and sagittal plane kinematics (peak and minimum joint angles, mean angular velocity (MAV) and range of motion (ROM)) during 10 deceleration trials preceded by $20 \mathrm{~m}$ runs at $50 \%$ effort $\left(3.61 \mathrm{~m} \cdot \mathrm{s}^{-1}\right)$, measured by $3 \mathrm{D}$ optical motion analysis (3DOMA) and IMU system. Validity between systems assessed using Pearson correlation coefficients $(r)$ and mean difference $( \pm 90 \%$ confidence limits $(C L))$ with effect size $(E S, \pm 90 \% \mathrm{CL})$. Magnitudes are presented for $r$ and $E S(m)$

\begin{tabular}{|c|c|c|c|c|c|c|c|c|c|}
\hline \multirow{2}{*}{ Variable } & \multirow{2}{*}{$n$} & 3DOMA & IMU & & \multirow{2}{*}{$m$} & Mean Difference & \multirow{2}{*}{$E S$} & \multirow{2}{*}{$m$} & \multirow{2}{*}{$\begin{array}{c}\text { Raw TEE } \\
( \pm 90 \% \mathrm{CL})\end{array}$} \\
\hline & & \multicolumn{2}{|c|}{ mean $(S D)$} & & & $( \pm 90 \% \mathrm{CL})$ & & & \\
\hline \multicolumn{10}{|c|}{ Temporal-spatial } \\
\hline GCT (s) & 33 & $0.24(0.04)$ & $0.25(0.04)$ & 0.87 & $\mathrm{VL}$ & 0.01 (0.00 to 0.01$)$ & $0.13(-0.02$ to 0.27$)$ & $T$ & $0.02(0.02$ to 0.02$)$ \\
\hline Foot-COM (m) & 28 & $0.39(0.06)$ & $0.40(0.05)$ & 0.80 & VL & $-0.02(-0.03$ to 0.01$)$ & $-0.33(-0.52$ to -0.14$)$ & $S$ & 0.03 (0.03 to 0.04$)$ \\
\hline \multicolumn{10}{|c|}{ Ankle } \\
\hline Peak dorsiflexion $\left({ }^{\circ}\right)$ & 33 & $13.9(5.4)$ & $15.6(4.7)$ & 0.06 & $S$ & $1.7(-0.4$ to 3.75$)$ & $-0.37(0.00$ to 0.74$)$ & $S$ & $5.5(4.5$ to 7.0$)$ \\
\hline Peak plantarflexion $\left({ }^{\circ}\right)$ & 33 & $15.1(3.6)$ & $4.2(5.4)$ & 0.45 & M & 10.9 (9.4 to 12.4$)$ & 3.00 (2.60 to 3.41$)$ & $V L$ & $3.2(2.7$ to 4.1$)$ \\
\hline $\operatorname{ROM}\left({ }^{\circ}\right)$ & 33 & $29.0(4.7)$ & $19.7(6.7)$ & 0.14 & S & $-9.3(-11.5$ to -7.0$)$ & $-1.61(-2.07$ to -1.15$)$ & $\mathrm{L}$ & $5.4(4.5$ to 6.9$)$ \\
\hline MAV dorsiflexion $\left({ }^{\circ} . \mathrm{s}^{-1}\right)$ & 33 & $229.3(71)$ & $146.3(84.2)$ & 0.39 & M & $-83.0(-108.5$ to -57.6$)$ & $-1.17(-1.53$ to -0.81$)$ & M & 66.4 (55.1 to 84.3$)$ \\
\hline $\operatorname{MAV}$ plantarflexion $\left({ }^{\circ} \cdot \mathrm{S}^{-1}\right)$ & 33 & $307.4(113.5)$ & $218.3(104.8)$ & 0.45 & $M$ & $-89.1(-123.0$ to -55.2$)$ & $-0.79(-1.08$ to -0.49$)$ & $M$ & 103.1 (85.6 to 130.7$)$ \\
\hline \multicolumn{10}{|c|}{ Knee } \\
\hline Minimum flexion $\left({ }^{\circ}\right)$ & 33 & $20.0(7.0)$ & $23.9(5.5)$ & 0.80 & $\mathrm{VL}$ & $-3.9(-5.2$ to -2.8$)$ & $-0.57(-0.75$ to -0.40$)$ & $S$ & 4.3 (3.5 to 5.4$)$ \\
\hline Peak flexion $\left({ }^{\circ}\right)$ & 33 & $93.1(5.4)$ & $93.6(7.4)$ & 0.69 & $\mathrm{~L}$ & $-0.5(-2.1$ to 1.1$)$ & $-0.09(-0.39$ to 0.20$)$ & $\mathrm{T}$ & $4.0(3.3$ to 5.0$)$ \\
\hline $\operatorname{ROM}\left({ }^{\circ}\right)$ & 33 & $73.1(9.4)$ & $69.6(10.7)$ & 0.93 & AP & $-3.5(-4.7$ to -2.3$)$ & $-0.37(-0.50$ to -0.24$)$ & $S$ & $3.6(3.0$ to 4.6$)$ \\
\hline $\operatorname{MAV}$ flexion $\left({ }^{\circ} \cdot \mathrm{s}^{-1}\right)$ & 33 & $325.0(76.3)$ & $303.2(73.0)$ & 0.98 & AP & $-21.8(-25.9$ to -17.8$)$ & $-0.29(-0.34$ to -0.23$)$ & $S$ & 13.8 (11.4 to 17.4$)$ \\
\hline \multicolumn{10}{|c|}{ Hip } \\
\hline Peak flexion $\left({ }^{\circ}\right)$ & 32 & $48.5(6.2)$ & $47.2(6.7)$ & 0.95 & AP & $-1.3(-1.9$ to -0.7$)$ & $-0.21(-0.31$ to -0.11$)$ & $S$ & $1.9(1.6$ to 2.4$)$ \\
\hline Peak extension $\left({ }^{\circ}\right)$ & 32 & $37.6(5.7)$ & $35.7(6.1)$ & 0.95 & AP & $-1.8(-2.4$ to -1.3$)$ & $-0.32(-0.42$ to -0.22$)$ & $S$ & 1.8 (1.5 to 2.3$)$ \\
\hline $\operatorname{ROM}\left({ }^{\circ}\right)$ & 32 & $10.9(3.2)$ & $11.5(3.8)$ & 0.83 & VL & $0.5(-0.1$ to 1.2$)$ & $0.17(-0.03$ to 0.36$)$ & $\mathrm{T}$ & $1.8(1.5$ to 2.3$)$ \\
\hline $\operatorname{MAV}$ flexion $\left({ }^{\circ} \cdot \mathrm{s}^{-1}\right)$ & 32 & $90.2(29.1)$ & $102.6(32.2)$ & 0.78 & VL & $12.4(6.3$ to 18.5$)$ & $0.43(0.22$ to 0.64$)$ & S & 18.4 (15.3 to 23.5$)$ \\
\hline MAV extension $\left({ }^{\circ} . \mathrm{S}^{-1}\right)$ & 32 & $74.1(42.0)$ & $85.1(43.4)$ & 0.65 & $\mathrm{~L}$ & $10.9(0.1$ to 21.7$)$ & $0.26(0.00$ to 0.52$)$ & S & 32.6 (27.0 to 41.5$)$ \\
\hline
\end{tabular}


At $50 \%$ effort on the approach ( $3.61 \mathrm{~m} . \mathrm{s}^{-1}$, see Table 1), measures of temporal-spatial parameters and most kinematics at the knee and hip obtained by Xsens IMU were valid as per criteria $(r \geq 0.7$ and ES < 0.6$)$. Largealmost perfect correlations and trivial-small ES were found in temporal-spatial parameters and kinematics at the knee and hip. Peak knee flexion and hip extension MAV were very close to meeting the criteria with large correlation and trivial-small ES ( $r=0.69$ and $-0.09 \mathrm{ES}, r=0.65$ and $0.26 \mathrm{ES}$, respectively). The ankle kinematics did not meet the criteria of validity due to small-moderate correlation coefficients and small-very large ES.

Table 2. Mean (standard deviation) of temporal-spatial parameters (Ground Contact Time (GCT) and anterior foot to centre of mass displacement (Foot-COM) and sagittal plane kinematics (peak and minimum joint angles, mean angular velocity (MAV) and range of motion (ROM)) during 10 deceleration trials preceded by $20 \mathrm{~m}$ runs at $75 \%$ effort $\left(5.81 \mathrm{~m} \cdot \mathrm{s}^{-1}\right)$, measured by $3 \mathrm{D}$ optical motion analysis (3DOMA) and IMU system. Validity between systems assessed using Pearson correlation coefficients $(r)$ and mean difference ( $\pm 90 \%$ confidence limits $(C L))$ with effect size $(E S, \pm 90 \% \mathrm{CL})$. Magnitudes are presented for $r$ and $E S(m)$

\begin{tabular}{|c|c|c|c|c|c|c|c|c|c|}
\hline \multirow{2}{*}{ Variable } & \multirow{2}{*}{$\mathrm{n}$} & 3DOMA & IMU & \multirow{2}{*}{$r$} & \multirow{2}{*}{$m$} & Mean Difference & \multirow{2}{*}{$E S$} & \multirow{2}{*}{$m$} & \multirow{2}{*}{$\begin{array}{c}\text { Raw TEE } \\
( \pm 90 \% \mathrm{CL})\end{array}$} \\
\hline & & \multicolumn{2}{|c|}{ mean $(S D)$} & & & $( \pm 90 \% \mathrm{CL})$ & & & \\
\hline \multicolumn{10}{|c|}{ Temporal-spatial } \\
\hline $\mathrm{GCT}(\mathrm{s})$ & 31 & $0.18(0.04)$ & $0.20(0.04)$ & 0.92 & AP & $0.02(0.02$ to 0.03$)$ & $0.48(0.36$ to 0.61$)$ & S & 0.02 (0.01 to 0.02 ) \\
\hline Foot-COM (m) & 26 & $0.40(0.13)$ & $0.42(0.05)$ & 0.84 & VL & $-0.02(-0.06$ to 0.01$)$ & $-0.18(-0.46$ to 0.11$)$ & $T$ & $0.03(0.02$ to 0.03$)$ \\
\hline \multicolumn{10}{|c|}{ Ankle } \\
\hline Peak Dorsiflexion $\left({ }^{\circ}\right)$ & 29 & $7.7(4.5)$ & $7.7(4.9)$ & 0.35 & M & $0.0(-1.7$ to 1.7$)$ & 0.00 (-0.38 to 0.38$)$ & $\mathrm{T}$ & 4.3 (3.49 to 5.50$)$ \\
\hline Peak Plantarflexion $\left({ }^{\circ}\right)$ & 29 & $22.8(6.5)$ & $11.5(6.8)$ & 0.66 & L & 11.3 (9.5 to 13.1$)$ & $1.73(1.46$ to 2.00$)$ & L & 5.0 (4.10 to 6.45$)$ \\
\hline $\operatorname{ROM}\left({ }^{\circ}\right)$ & 29 & $30.6(4.7)$ & $19.2(7.3)$ & 0.60 & L & $-10.9(-12.7$ to -9.0$)$ & $-2.33(-2.73$ to -1.93$)$ & VL & 3.8 (3.13 to 4.93$)$ \\
\hline MAV Dorsiflexion $\left({ }^{\circ} . s^{-1}\right)$ & 29 & $364.3(78.7)$ & $194.5(99.8)$ & 0.22 & $T$ & $-169.8(-205.3$ to 134.2$)$ & $-2.16(-2.61$ to -1.71$)$ & VL & 77.0 (62.8 to 100.8$)$ \\
\hline MAV Plantarflexion $\left({ }^{\circ} . \mathrm{s}^{-1}\right)$ & 29 & $428.2(259.6)$ & $247.5(184.9)$ & 0.28 & $S$ & $-180.7(-271.6$ to -89.8$)$ & $-0.70(-1.05$ to -0.35$)$ & M & 161.6 (130.6 to 214.1$)$ \\
\hline \multicolumn{10}{|c|}{ Knee } \\
\hline Minimum Flexion $\left({ }^{\circ}\right)$ & 30 & $17.0(4.7)$ & $19.8(5.1)$ & 0.28 & $S$ & $-2.8(-4.6$ to -1.0$)$ & $-0.60(-0.99$ to -0.22$)$ & M & 4.6 (3.8 to 5.9$)$ \\
\hline Peak Flexion $\left({ }^{\circ}\right)$ & 30 & $95.5(5.3)$ & $97.1(8.7)$ & 0.53 & L & $-1.6(-3.9$ to 0.7$)$ & $-0.31(-0.74$ to 0.13$)$ & $S$ & 4.6 (3.7 to 5.9$])$ \\
\hline $\operatorname{ROM}\left({ }^{\circ}\right)$ & 30 & $78.5(5.2)$ & $77.3(6.9)$ & 0.90 & VL & $-1.2(-2.8$ to -0.4$)$ & $-0.22(-0.41$ to -0.04$)$ & $S$ & 2.3 (1.9 to 3.0$)$ \\
\hline MAV Flexion $\left({ }^{\circ} \cdot s^{-1}\right)$ & 30 & $461.6(105.1)$ & $417.6(88.7)$ & 0.93 & AP & $-44.1(-56.3$ to -31.8$)$ & $-0.42-(-0.54$ to -0.30$)$ & $S$ & 38.3 (31.4 to 49.5$)$ \\
\hline \multicolumn{10}{|c|}{ Hip } \\
\hline Flexion $\left({ }^{\circ}\right)$ & 28 & $42.5(4.3)$ & $44.8(9.5)$ & 0.61 & L & $2.3(-0.2$ to 4.8$)$ & $0.54(-0.04$ to 1.1$)$ & $S$ & 3.4 (2.8 to 4.5$)$ \\
\hline Extension $\left({ }^{\circ}\right)$ & 28 & $34.0(5.4)$ & $31.4(7.5)$ & 0.61 & L & $-2.6(-4.5$ to -0.6$)$ & $-0.47(-0.83$ to -0.12$)$ & $S$ & 4.3 (3.6 to 5.6$)$ \\
\hline $\operatorname{ROM}\left({ }^{\circ}\right)$ & 28 & $8.5(2.9)$ & $13.3(5.2)$ & 0.43 & M & 4.8 (3.3 to 6.4$)$ & 1.68 (1.15 to 2.21$)$ & L & 2.7 (2.2 to 3.5$)$ \\
\hline MAV Flexion $\left({ }^{\circ} \cdot s^{-1}\right)$ & 28 & $128.7(114.0)$ & $151.0(63.8)$ & -0.05 & $\mathrm{~T}$ & $22.3(-20.69$ to 65.36$)$ & $0.20(-0.18$ to 0.57$)$ & $S$ & 116.0 (94.9 to 150.8$)$ \\
\hline MAV Extension $\left({ }^{\circ} . \mathrm{s}^{-1}\right)$ & 25 & $137.4(95.6)$ & $151.0(81.3)$ & 0.01 & $\mathrm{~T}$ & $13.6(-29.11$ to 56.30$)$ & $0.14(-0.30$ to 0.59$)$ & $T$ & 97.65 (79.0 to 129.4$)$ \\
\hline
\end{tabular}

Following an approach at $75 \%$ effort (5.81 m.s ${ }^{-1}$, see Table 2), the temporal-spatial parameters, knee ROM and knee flexion MAV obtained by Xsens IMU met the criteria for validity with very large-almost perfect correlation coefficients and trivial-small ES. Other measures of knee kinematics demonstrated small-moderate ES but were not were valid due to small-large correlations. Likewise, only trivial-large correlations were found in other measures at the hip with trivial-small ES. Hip flexion and extension were close to meeting the validity criteria with large correlation ( $r=0.61)$ and small ES $(0.54$ and -0.47 , respectively). None of the ankle kinematics met the criteria for validity and were attributed to trivial-large correlations and trivial-very large ES. 
Table 3. Mean (standard deviation) of temporal-spatial parameters (Ground Contact Time (GCT) and posterior foot to centre of mass displacement (Foot-COM) and sagittal plane kinematics (peak and minimum joint angles, mean angular velocity (MAV) and range of motion (ROM)) during 10 deceleration trials preceded by $20 \mathrm{~m}$ runs at $100 \%$ effort $\left(6.45 \mathrm{~m}^{-\mathrm{s}^{-1}}\right)$, measured by 3D optical motion analysis (3DOMA) and IMU system. Validity between systems assessed using Pearson correlation coefficients $(r)$ and mean difference $( \pm 90 \%$ confidence limits $(C L))$ with effect size $(E S, \pm 90 \% \mathrm{CL})$. Magnitudes are presented for $r$ and $E S(m)$

\begin{tabular}{|c|c|c|c|c|c|c|c|c|c|}
\hline \multirow{2}{*}{ Variable } & \multirow{2}{*}{$\mathrm{n}$} & 3DOMA & IMU & & \multirow{2}{*}{$m$} & Mean Difference & \multirow{2}{*}{$E S$} & \multirow{2}{*}{$m$} & \multirow{2}{*}{$\begin{array}{c}\text { Raw TEE } \\
( \pm 90 \% \mathrm{CL})\end{array}$} \\
\hline & & mean $(S D)$ & mean $(\mathrm{SD})$ & & & $( \pm 90 \% \mathrm{CL})$ & & & \\
\hline \multicolumn{10}{|c|}{ Temporal-spatial } \\
\hline GCT (s) & 68 & $0.19(0.04)$ & $0.19(0.04)$ & 0.86 & $\mathrm{VL}$ & 0.00 (0.00 to 0.01$)$ & $0.07(-0.03$ to 0.18$)$ & $\mathrm{T}$ & 0.02 (0.02 to 0.03$)$ \\
\hline Foot-COM (m) & 52 & $0.44(0.06)$ & $0.45(0.06)$ & 0.39 & $S$ & $0.01(-0.04$ to -0.05$)$ & $0.21(-0.06$ to 0.48$)$ & S & $0.18(0.16$ to 0.22$)$ \\
\hline \multicolumn{10}{|c|}{ Ankle } \\
\hline Peak Dorsiflexion $\left({ }^{\circ}\right)$ & 68 & $5.7(4.8)$ & $2.1(5.7)$ & 0.41 & $M$ & $-3.6(-4.7$ to -2.4$)$ & $-0.74(-0.98$ to -0.49$)$ & M & 4.5 (3.9 to 5.2$)$ \\
\hline Peak Plantarflexion $\left({ }^{\circ}\right)$ & 68 & $25.3(3.5)$ & $20.6(5.3)$ & 0.48 & M & 4.7 (3.7 to 5.6$)$ & 1.35 (1.07 to 1.63$)$ & L & 3.1 (2.7 to 3.6 ) \\
\hline $\operatorname{ROM}\left({ }^{\circ}\right)$ & 68 & $31.0(4.5)$ & $22.7(7.4)$ & 0.53 & L & $-8.3(-9.8$ to -7.2$)$ & $-1.91(2.20$ to -1.62$)$ & L & 3.8 (3.3 to 4.4$)$ \\
\hline MAV Dorsiflexion $\left({ }^{\circ} . \mathrm{s}^{-1}\right)$ & 68 & 376.5 (99.9) & $225.7(114.3)$ & 0.27 & $S$ & $-150.8(-177.0$ to -124.6$)$ & $-1.51(-1.77$ to -1.25$)$ & $\mathrm{L}$ & 96.8 (84.8 to 113.2$)$ \\
\hline MAV Plantarflexion $\left({ }^{\circ} . s^{-1}\right)$ & 68 & $483.7(130.1)$ & $275.8(188.4)$ & 0.44 & M & $-207.9(-224.3$ to -171.6$)$ & $-1.60(-1.88$ to -1.32$)$ & L & 117.8 (102.9 to 138.2$)$ \\
\hline \multicolumn{10}{|c|}{ Knee } \\
\hline Minimum Flexion $\left({ }^{\circ}\right)$ & 69 & $19.0(8.7)$ & $15.2(4.3)$ & 0.45 & $M$ & $3.8(2.2$ to 5.3$)$ & 0.44 (0.26 to 0.62$)$ & $S$ & 7.8 (6.8 to 9.1$)$ \\
\hline Peak Flexion $\left({ }^{\circ}\right)$ & 69 & $95.5(9.4)$ & $88.7(10.6)$ & 0.45 & M & $6.8(-8.9$ to -4.7$)$ & 0.72 (0.50 to 0.95$)$ & M & 8.5 (7.4 to 9.9$)$ \\
\hline $\operatorname{ROM}\left({ }^{\circ}\right)$ & 69 & $76.5(9.0)$ & $73.5(10.4)$ & 0.29 & $S$ & $-3.0(-5.7$ to -0.3$)$ & $-0.46(-0.72$ to -0.20$)$ & S & $8.7(7.6$ to 10.1$)$ \\
\hline MAV Flexion $\left({ }^{\circ} \cdot s^{-1}\right)$ & 69 & $469.4(104.7)$ & $441.2(97.2)$ & 0.84 & VL & $-28.2(-39.7$ to -16.8$)$ & $-0.27(-0.38$ to -0.16$)$ & S & 56.9 (49.9 to 66.4$)$ \\
\hline \multicolumn{10}{|c|}{ Hip } \\
\hline Peak flexion $\left({ }^{\circ}\right)$ & 69 & $42.8(4.8)$ & $37.9(5.7)$ & 0.74 & VL & $-4.9(-5.7$ to -4.2$)$ & $-1.03(-1.19$ to -0.87$)$ & M & 3.2 (2.8 to 3.8$)$ \\
\hline Peak extension $\left({ }^{\circ}\right)$ & 69 & $32.3(4.7)$ & $23.1(6.6)$ & 0.65 & $L$ & $-8.8(-9.86$ to -7.81$)$ & $-1.94(-2.18$ to -1.69$)$ & L & 3.7 (3.2 to 4.3$)$ \\
\hline $\operatorname{ROM}\left({ }^{\circ}\right)$ & 69 & $10.5(3.4)$ & $14.8(5.8)$ & 0.35 & M & 4.3 (3.1 to 5.4$)$ & 1.37 (0.97 to 1.78$)$ & L & 3.2 (2.8 to 3.8 ) \\
\hline MAV Flexion $\left({ }^{\circ} \cdot s^{-1}\right)$ & 68 & $168.0(126.5)$ & $174.5(62.0)$ & 0.09 & $\mathrm{~T}$ & $6.5(-21.1$ to 34.0$)$ & $0.05(-0.17$ to 0.27$)$ & $\mathrm{T}$ & $127.0(11.2$ to 148.4$)$ \\
\hline MAV Extension $\left({ }^{\circ} \cdot \mathrm{s}^{-1}\right)$ & 69 & $121.2(77.5)$ & $159.7(84.9)$ & 0.37 & M & 40.6 (21.9 to 59.3$)$ & $0.50(0.27$ to 0.73$)$ & $S$ & 75.6 (66.3 to 88.3 ) \\
\hline
\end{tabular}

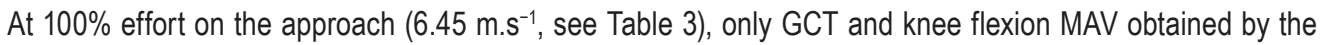
Xsens IMU system met the criteria for validity with very large correlations and trivial-small ES. Other kinematics at the knee and hip demonstrated small-moderate correlations with small-moderate ES and trivial-very large with trivial-large ES, respectively. Ankle kinematics did not meet the criteria for validity due to small-large correlations and moderate-large ES.

\section{Discussion}

This study sought to assess the validity of Xsens IMU system to measure temporal-spatial and lower-limb joint kinematic measures captured during a maximal linear deceleration, preceded by $20 \mathrm{~m}$ runs at $50 \%, 75 \%$ and $100 \%$ effort, when compared with 3DOMA. The key findings from this study were that during deceleration phase, Xsens IMU system had valid measures of: (1) knee and hip sagittal plane kinematics and temporal-spatial parameters from low approach velocities (3.61 m.s ${ }^{-1}, 50 \%$ effort), (2) knee flexion MAV, knee ROM and temporal spatial parameters from moderate approach velocity (5.81 m.s. $\mathrm{s}^{-1}, 75 \%$ effort), and (3) only GCT, knee flexion MAV from higher approach velocity (6.45 m.s $\mathrm{s}^{-1}, 100 \%$ effort). None of the ankle kinematics derived from Xsens IMU met the criteria of validity. 
The results of this study are comparable to other studies on the validity of Xsens IMU to measure temporalspatial parameters and joint kinematics during sport and physical activities. In this study, the hip and knee kinematics demonstrated large to almost perfect agreement $(r=0.69-0.98$ and $0.5-0.95)$, trivial to small ES $(-0.09$ to -0.57 and $-0.32-0.43$ ) and comparably low typical error (TEE $=1.8-1.9^{\circ}$ and $\left.3.6-4.3^{\circ}\right)$ when decelerating from low intensity running (50\% efforts). Similarly, strong agreement between Xsens and 3DOMA $(r>0.96)$ and similar estimates of the error associated with Xsens measurements of the hip $\left(1.8-5.2^{\circ}\right)$ and knee $\left(1.5-5.1^{\circ}\right)$ kinematics were found during walking (Ferrari et al., 2010; Seel et al., 2014; Zhang et al., 2013). Wouda et al. (2018) also reported strong agreement $(r>0.98)$ between Xsens and 3DOMA at the hip and knee during treadmill running at different speeds, however when compared with our study, larger errors were observed (mean difference $<18.1^{\circ}$ at the hip and $<10.2^{\circ}$ at knee). Krüger and Edelmann-Nusser (2009) also reported strong agreement $(r=0.77-0.96)$ and errors of less than $5^{\circ}$ for knee angles during a snowboarding turn, when compared with video analysis. When compared with the present study, smaller measurement error has been shown at the knee (peak flexion: $0.8^{\circ}$, peak extension: $1.4^{\circ}$ and mean angular velocity: $14^{\circ} . \mathrm{s}^{-1}$ ) and hip (hip extension: $0.5^{\circ}$ ) during a kicking action (Blair et al., 2018). Such high validity might be attributable to a simpler task of kicking, relative to deceleration, which involves many co-ordinated steps in quick succession.

As approach velocity increased $(75 \%-100 \%)$, the agreement between kinematic measures were weak at the hip ( $r=0.43-0.61$ and $0.35-0.74$, respectively) and reduced at the knee ( $r=0.28-0.93$ and $0.29-0.45$, respectively) and typical error increased at the hip $\left(2.7-4.3^{\circ}\right.$ and $3.2-3.7^{\circ}$ respectively) and knee $\left(2.3-4.6^{\circ}\right.$ and $7.8-8.7^{\circ}$, respectively) when compared with $50 \%$ effort. Also, the mean difference and ES increased, which suggests an increased systematic bias as intensity increased, hence most Xsens IMU measurements were not valid at higher intensities. Interestingly, knee flexion MAV was valid following $75 \%$ and $100 \%$ efforts. Rapid knee flexion during deceleration is a key indicator of injury during deceleration (Cochrane et al., 2007) and therefore this measure could be useful for practitioners, however with greater approach velocity typical error increased from 38.3 to $56.9^{\circ} \cdot \mathrm{s}^{-1}$. Similarly, an increase in joint kinematic error was reported as gait speed increased (0.6-5.0 $0^{\circ}$; Ferrari et al., 2010) and as segment velocity increased during a ball kicking action (Blair et al., 2018). The increased error with speed of movement has been attributed to the inertial sensor data gleaned from IMU, which can be compromised when sudden changes in acceleration are experienced (Fleron et al., 2019). Erroneous accelerations in IMU data might be exacerbated by greater skin and muscle movement underlying the IMUs during deceleration from faster approach velocities due to greater impact forces applied to the lower limb when compared with deceleration from slower approach velocities. Also, Xsens uses Kalman filters to fuse the IMU data, however Kalman filtering is limited during faster movements, especially from high to low segment acceleration (Adesida et al., 2019; Blair et al., 2018; Ferrari et al., 2010). Sudden changes in segmental acceleration are characteristic of deceleration and are greater in more intense decelerations, thus potentially compromising the measurement of most kinematic measures during deceleration from higher intensity running (5.81 m.s $\mathrm{s}^{-1}-6.45 \mathrm{~m} . \mathrm{s}^{-1}, 75 \%-100 \%$ effort).

Regardless of effort on the approach, ankle kinematics during linear deceleration did not meet the criteria for validity. Mixed reports of the validity of ankle measurement by IMU has been reported previously (Ferrari et al., 2010; Robert-Lachaine, Mecheri, Larue, Plamondon, 2017; Seel et al., 2014). The error of Xsens at the ankle has been reported to be low in the sagittal plane during walking (Ferrari et al.: $r>0.99$ and $1.1-1.4^{\circ}$; Seel et al.: $1.6^{\circ}$ ) which surpasses the validity of sagittal plane ankle measures found in this study of $3.1-5.5^{\circ}$, however others have reported weaker validity at the ankle which are somewhat comparable with this study. During treadmill running, 
Wouda et al. (2018) observed strong agreement in dorsi and plantarflexion $(r>0.97)$, however mean difference and error was larger $\left(>7.58^{\circ}\right.$ and $>8.1^{\circ}$, respectively) than in this study. There are several reasons for poorer validity at the ankle. The highest segment velocities are observed at the distal end of limbs and therefore the foot might be affected by the aforementioned Kalman filter, thus introducing error to the ankle, even during decelerations from low intensity running ( $50 \%$ efforts). The Kalman filter was purported to be responsible for sudden changes in orientation of body segments in the Xsens system during walking, most of which occurred at the ankle (Ferrari et al., 2010). This might account for the lack of valid measures at the ankle; however, the authors do not have definitive evidence of whether this was the case in this study. Another source of error could be attributed to the gyroscopes in the IMUs which are sensitive to vibrations when the foot contacts the ground. During deceleration, large impact and eccentric braking forces are observed upon contact with the ground (Hewit et al., 2011) and might introduce vibration noise to the IMU signal which was likely amplified as the intensity of deceleration increased.

The point of application of force relative to the COM is also important for deceleration performance and this study suggests that foot-COM measures lack validity during deceleration from high intensity running $\left(6.45 \mathrm{~m} . \mathrm{s}^{-1}\right.$, $100 \%$ effort) with large error observed (TEE $0.18 \mathrm{~m}$ ) but is valid during deceleration preceded by low and moderate running (3.61-5.81 m.s ${ }^{-1}, 50-75 \%$ effort). Despite this, Xsens IMU system can be used to determine initial contact and toe-off and subsequently provide valid measures of GCT during linear decelerations. Ground contact time is an important determinant of deceleration as braking forces can only be applied during contact (Hewit et al., 2011). Using the methods detailed in this study, practitioners could use Xsens IMU to assess GCT during submaximal and maximal linear decelerations.

There are some limitations to acknowledge for this study. Movement artefact from underlying muscle and skin could result in displacements or translation of the IMU along the segment longitudinal axis which could affect segment orientation and subsequent calculation of joint angles. The Xsens IMU units were strapped firmly to the participant and the filtering process by Xsens might remove such movement artefact. Likewise, the 3DOMA might also be subject to movement artefact on the reflective markers, however such movements are reduced by appropriate filtering. The duration of between task and calibration of IMU system has been reported to introduce drift and error into the IMU data (Robert-Lachaine et al., 2017; Roetenberg, Luinge, Slycke, 2009). The participant undertook the calibration for both systems regularly to reduce the likelihood of drift. Fatigue of the participant from repeated ADA trials might have affected their ability to maintain consistent approach velocities for each perceived effort, especially in the repeated maximal ADA trials. The duration of recovery between the ADA trials ranged from 3-5 min, which was considered sufficient for the participant, a trained and competitive soccer player, to be rested and relatively small standard deviations were found for each approach velocity $\left(0.33,0.14\right.$ and $0.24 \mathrm{~m}_{\mathrm{s}} \mathrm{s}^{-1}$ at 50 , 75 and 100\% efforts, respectively). However, an objective physiological measure, such as heart rate monitoring, could have been used to confirm if the participant was indeed rested prior to each ADA trial. It has been noted that biomechanical model definitions might be responsible for differences and therefore caution must be taken when directly comparing joint angles from IMU and 3DOMA (Robert-Lachaine et al., 2017). The differences in the model were limited since both models were multi-segmental and the IMU model was closely aligned with anatomical landmarks used in 3DOMA.

In conclusion, Xsens IMU offers valid measures of joint kinematics of the hip and knee during linear decelerations when the approach velocity is low (3.61 m.s $\mathrm{s}^{-1}, 50 \%$ effort) but limited to valid measures of knee flexion MAV when decelerating from higher approach velocities $\left(5.81-6.45 \mathrm{~m} . \mathrm{s}^{-1}, 75-100 \%\right.$ efforts). Regardless 
of approach velocity, Xsens IMU does not provide valid measurement of ankle kinematics during deceleration. Basic temporal-spatial parameters can be measured using Xsens IMU during deceleration following all approach velocities, except for foot-COM following 100\% effort. Therefore, coaches and practitioners could use Xsens IMU to assess temporal-spatial parameters and kinematics of the hip and knee from low approach velocities $\left(>3.61 \mathrm{~m} . \mathrm{s}^{-1}\right)$ and limited knee kinematics at higher approach velocities. Despite the drawbacks of 3DOMA, it is recommended that 3DOMA should be considered for the assessment of decelerations from higher approach velocities.

\section{References}

Abt, G., Siegler, J.C., Akubat, I., Castagna, C. (2011). The effects of a constant sprint-to-rest ratio and recovery mode on repeated sprint performance. The Journal of Strength \& Conditioning Research, 25 (6), 1695-1702. DOI: 10.1519/JSC.0b013e3181dbdc06.

Adesida, Y., Papi, E., McGregor, A.H. (2019). Exploring the role of wearable technology in sport kinematics and kinetics: A systematic review. Sensors, 19, 1597. DOI: 10.3390/s19071597.

Balsom, P.D., Seger, J.Y., Sjödin, B., Ekblom, B. (1992). Physiological responses to maximal intensity intermittent exercise. European Journal of Applied Physiology and Occupational Physiology, 65 (2), 144-149. DOI: 10.1007/BF0070572.

Blair, S., Duthie, G., Robertson, S., Hopkins, W., Ball, K. (2018). Concurrent validation of an inertial measurement system to quantify kicking biomechanics in four football codes. Journal of Biomechanics, 73 (1), 24-32. DOI: 10.1016/j.jbiomech.2018.03.031.

Bloomfield, J., Polman, R., O'Donoghue, P. (2008). Deceleration and turning movements performed during FA Premier League soccer matches. In: T. Reilly, F. Korkusuz (eds.), Science and Football Vl; the Proceedings of the Sixth World Congress on Science and Football (pp. 174-181). Routledge.

Buchheit, M., Gray, A., Morin, J.-B. (2015). Assessing stride variables and vertical stiffness with GPS-embedded accelerometers: Preliminary insights for the monitoring of neuromuscular fatigue on the field. Journal of Sports Science \& Medicine, 14 (4), 698-701.

Camomilla, V., Bergamini, E., Fantozzi, S., Vannozzi, G. (2018). Trends supporting the in-field use of wearable inertial sensors for sport performance evaluation: A systematic review. Sensors, 18, 873. DOI: 10.3390/s18030873.

Carson, H.J., Collins, D., Richards, J. (2014). Intra-individual movement variability during skill transitions: A useful marker? European Journal of Sport Science, 14 (4), 327-336. DOI: 10.1080/17461391.2013.814714.

Cesar, G.M., Sigward, S.M. (2015). Dynamic stability during running gait termination: Differences in strategies between children and adults to control forward momentum. Human Movement Science, 43 (1), 138-145. DOI: 10.1016/j.humov.2015.08.005.

Cesar, G.M., Sigward, S.M. (2016). Dynamic stability during running gait termination: Predictors for successful control of forward momentum in children and adults. Human Movement Science, 48 (1), 37-43. DOI: 10.1016/j.humov.2016.03.014.

Chaouachi, A., Manzi, V., Chaalali, A., Wong, P., Chamari, K., Castagna, C. (2012). Determinants analysis of change-of-direction ability in elite soccer players. Journal of Strength \& Conditioning Research, 26, 2667-2676. DOI: 10.1519/JSC.0b013e318242f97a.

Cochrane, J.L., Lloyd, D.G., Buttfield, A., Seward, H., McGivern, J. (2007). Characteristics of anterior cruciate ligament injuries in Australian football. Journal of Science and Medicine in Sport, 10 (2), 96-104. DOI: 10.1016/j.jsams.2006.05.015.

Coleman, A.E. (2001). Target and recovery times for speed training in baseball. Strength \& Conditioning Journal, 23 (6), 7-8.

Dalen, T., Ingebrigtsen, J., Ettema, G., Hjelde, G.H., Wisloff, U. (2016). Player load, acceleration, and deceleration during fortyfive competitive matches of elite soccer. Journal of Strength \& Conditioning Research, 30 (2), 351-359. DOI: 10.1519/ JSC. 0000000000001063.

Dos'Santos, T., Thomas, C., Comfort, P., Jones, P.A. (2018). The effect of angle and velocity on change of direction biomechanics: An angle-velocity trade-off. Sports Medicine, 48 (10), 2235-2253. DOI: 10.1007/s40279-018-0968-3.

Ferrari, A., Cutti, A.G., Garofalo, P., Raggi, M., Heijboer, M., Cappello, A., Davalli, A. (2010). First in vivo assessment of "Outwalk": A novel protocol for clinical gait analysis based on inertial and magnetic sensors. Medical \& Biological Engineering \& Computing, 48, 1-15. DOI: 10.1007/s11517-009-0544-y.

Fleron, M.K., Ubbesen, N.C.H., Battistella, F., Dejtiar, D.L., Oliveira, A.S. (2019). Accuracy between optical and inertial motion capture systems for assessing trunk speed during preferred gait and transition periods. Sports Biomechanics, 18 (4), 366-377. DOI: 10.1080/14763141.2017.1409259.

Green, B.S., Blake, C., Caulfield, B.M. (2011). A comparison of cutting technique performance in rugby union players. Journal of Strength \& Conditioning Research, 25 (10), 2668-2680. DOI: 10.1519/JSC.0b013e318207ed2a. 
Harper, D., Jordan, A., Kiely, J. (2021). Relationships between eccentric and concentric knee strength capacities and maximal linear deceleration ability in male academy soccer players. Journal of Strength \& Conditioning Research, 35 (2), 465-472. DOI: 10.1519/JSC.0000000000002739.

Harper, D., Kiely, J. (2018). Damaging nature of decelerations: Do we adequately prepare players? BMJ Open Sport and Exercise Medicine, 4, e000379. DOI: 10.1136/bmjsem-2018-000379.

Hewit, J., Cronin, J., Button, C., Hume, P. (2011). Understanding deceleration in sport. Strength and Conditioning Journal, 33 (1), $47-52$. DOI: 10.1519/SSC.0b013e3181fbd62c.

Hopkins, W.G. (2002). A scale of magnitudes for effect statistics. A New View of Statistics, 502, 411. Retrieved from: https://sportsci. org/resource/stats/effectmag.html.

Kovacs, M.S., Roetert, E.P., Ellenbecker, T.S. (2008). Efficient deceleration: The forgotten factor in tennis-specific training. Strength and Conditioning Journal, 30 (6), 58-69. DOI: 10.1519/SSC.0b013e31818e5fbc.

Krüger, A., Edelmann-Nusser, J. (2009). Biomechanical analysis in freestyle snowboarding: Application of a full-body inertial measurement system and a bilateral insole measurement system. Sports Technology, 2 (1-2), 17-23. DOI: 10.1002/jst.89.

Laudanski, A., Brouwer, B., Li, Q. (2013). Measurement of lower limb joint kinematics using inertial sensors during stair ascent and descent in healthy older adults and stroke survivors. Journal of Healthcare Engineering, 4 (4), 555-576. DOI: 10.1260/2040-2295.4.4.555.

Mara, J.K., Thompson, K.G., Pumpa, K.L., Morgan, S. (2017). The acceleration and deceleration profiles of elite female soccer players during competitive matches. Journal of Science and Medicine in Sport, 20 (9), 867-872. DOI: 10.1016/j.jsams.2016.12.078.

Nedergaard, N.J., Kersting, U., Lake, M. (2014). Using accelerometry to quantify deceleration during a high-intensity soccer turning manoeuvre. Journal of Sports Sciences, 32 (20), 1897-1905. DOI: 10.1080/02640414.2014.965190.

Reenalda, J., Maartens, E., Homan, L., Buurke, J.J. (2016). Continuous three dimensional analysis of running mechanics during a marathon by means of inertial magnetic measurement units to objectify changes in running mechanics. Journal of Biomechanics, 49 (14), 3362-3367. DOI: 10.1016/j.jbiomech.2016.08.032.

Robert-Lachaine, X., Mecheri, H., Larue, C., Plamondon, A. (2017). Validation of inertial measurement units with an optoelectronic system for whole-body motion analysis. Medical and Biological Engineering and Computing, 55 (4), 609-619. DOI: 10.1016/j. jbiomech.2016.08.032.

Roetenberg, D., Luinge, H., Slycke, P. (2009). Xsens MVN: Full 6DOF human motion tracking using miniature inertial sensors. Technical report. Xsens Technologies B.V.

Russell, M., Sparkes, W., Northeast, J., Cook, C.J., Love, T.D., Bracken, R.M., Kilduff, L.P. (2016). Changes in acceleration and deceleration capacity throughout professional soccer match-play. Journal of Strength \& Conditioning Research, 30 (10), 28392844. DOI: $10.1519 / J S C .0000000000000805$.

Seel, T., Raisch, J., Schauer, T. (2014). IMU-based joint angle measurement for gait analysis. Sensors, 14 (4), 6891-6909. DOI: 10.3390/s140406891.

Spencer, M., Bishop, D., Dawson, B., Goodman, C. (2005). Physiological and metabolic responses of repeated-sprint activities. Sports Medicine, 35 (12), 1025-1044. DOI: 10.2165/00007256-200535120-00003.

Spiteri, T., Nimphius, S., Hart, N.H., Specos, C., Sheppard, J.M., Newton, R.U. (2014). Contribution of strength characteristics to change of direction and agility performance in female basketball athletes. Journal of Strength \& Conditioning Research, 28 (9), 2415-2423. DOI: 10.1519/JSC.0000000000000547.

Wouda, F.J., Giuberti, M., Bellusci, G., Maartens, E., Reenalda, J., Van Beijnum, B.F., Veltink, P.H. (2018). On the validity of different motion capture technologies for the analysis of running. Paper presented at the 7th IEEE/RAS-EMBS International Conference on Biomedical Robotics and Biomechatronics (Biorob), Enschede.

Zhang, J.-T., Novak, A.C, Brouwer, B., Li, Q. (2013). Concurrent validation of Xsens MVN measurement of lower limb joint angular kinematics. Physiological Measurement, 34 (8), N63-N69. DOI: 10.1088/0967-3334/34/8/N63.

Cite this article aS: Jordan, A.R., Carson, H.J., Wilkie, B., Harper, D.J. (2021). Validity of an Inertial Measurement Unit System to Assess Lower-limb Kinematics during a Maximal Linear Deceleration. Central European Journal of Sport Sciences and Medicine, 1 (33), 5-16. DOI: 10.18276/cej.2021.1-01. 